ÉGYPTE

monde arabe

\section{Égypte/Monde arabe}

$22 \mid 1995$

Géographies de l'Égypte 1

\title{
Cohérence et cohésion d'un espace. Une présentation de la ville ancienne du Caire
}

\section{Anna Madœuf}

\section{(2) OpenEdition}

\section{Journals}

Édition électronique

URL : https://journals.openedition.org/ema/605

DOI : $10.4000 /$ ema.605

ISSN : 2090-7273

\section{Éditeur}

CEDEJ - Centre d'études et de documentation économiques juridiques et sociales

\section{Édition imprimée}

Date de publication : 30 juin 1995

Pagination : $97-121$

ISSN : 1110-5097

\section{Référence électronique}

Anna Madœuf, «Cohérence et cohésion d'un espace. Une présentation de la ville ancienne du Caire ». Égypte/Monde arabe [En ligne], 22 | 1995, mis en ligne le 08 juillet 2008, consulté le 07 juillet 2022. URL : http://journals.openedition.org/ema/605 ; DOI : https://doi.org/10.4000/ema.605

Ce document a été généré automatiquement le 7 juillet 2022.

Tous droits réservés 


\title{
Cohérence et cohésion d'un espace. Une présentation de la ville ancienne du Caire
}

\author{
Anna Madœuf
}

$1 \mathrm{Au}$ Caire ${ }^{1}$, le terme de wast al-balad («centre-ville ») renvoie à un espace précis - la ville «moderne» du XIx $x^{e}$ siècle, fondée par le khédive Ismâ'îl; quant à la ville dite "ancienne", elle continue à être désignée par des termes aussi divers que ville « orientale ", « islamique », « médiévale », « fatimide ", « historique », « Vieux-Caire »bien que cette appellation se réfère plutôt au quartier copte, Masr al-Qadîma - ou «vieille ville». En ce qui concerne cette dernière, Mona Zakariya parle encore de « zones traditionnelles », Günter Meyer de «old quarters of central Cairo » et Philippe Panerai suggère la notion de «vieille-ville moderne ». Quant au concept de «médina », remarque Mercedes Volait, il ne fait « pas - ou plus? - partie du vocabulaire courant, alors qu'il est resté d'usage commun dans d'autres pays de la région $»^{2}$. Les documents officiels et les médias nomment cet ensemble al-Qâhira al-islâmiyya wa-l-fâtimiyya - Le Caire islamique et fatimide. Quant aux Cairotes, ils ne le désignent pas, au quotidien, par un nom générique, mais par des noms de monuments - «al-Azhar» ou «alHusayn », le nom des deux grandes mosquées qui dominent le quartier - ou de lieux « Khan al-Khalîlî », «al-Ghûriyya » ou encore "akhir Mûski» («au bout de la rue du Mûski »).

2 Comment appréhender la ville ancienne, vaste ensemble de quartiers bien identifiés mais espace sans nom propre, résultat d'histoires passées et contemporaines, de superpositions et de stratifications urbaines? Peut-être faut-il tout d'abord rappeler, «à qui s'intéresse à la réalité d'aujourd'hui, que le passé qu'il rencontre dans le présent à travers ce qui en survit, menacé, nié ou/et idéalisé, s'est lui-même produit, à des rythmes et selon des durées diverses, selon des héritages et des ruptures, des continuités et des changements » (Depaule, 1987). 
3 Ce qui est une facette de la ville actuelle a été la ville, mais surtout, continue à l'être. Espace qui fut un et unique, aujourd'hui « cœur de mégapole », la ville ancienne, sans cesse renouvelée, est toujours en quête de définition.

$4 \mathrm{Si}$ « le paysage naît de la rencontre d'un espace et d'un regard " ${ }^{3}$, alors nous tenterons, à partir d'une lecture de travaux portant sur la ville ancienne, de tracer l'esquisse d'un paysage commun en croisant des caractéristiques et des éléments de cet espace, mis à jour à des échelles différentes, depuis la fin des années 60 jusqu'à nos jours, par des historiens, architectes, géographes, anthropologues, sociologues et urbanistes. Des aspects constitutifs des représentations de la ville ancienne seront également empruntés à la littérature égyptienne contemporaine.

5 Cette analyse sera généraliste; la transversalité des thèmes évoqués ne sera qu'effleurée. Le concept de "formation socio-spatiale » élaboré par Guy Di Méo sera utilisé comme méthode d'approche globalisante. Il ne s'agit pas de chercher un modèle parfait, puisque "les ébauches, les esquisses inachevées sont plus nombreuses que les formations bien construites et indiscutables. La formation socio-spatiale s'avère beaucoup plus une tendance organisationnelle de l'espace social qu'une réalité objective parfaitement cernée »; il s'agit plutôt d'utiliser une méthode fournissant des indications, des pistes, un cadre d'analyse :

La recherche d'une instance géographique à la base de toute formation sociospatiale consistera à repérer les superpositions et les interférences les plus significatives des dispositifs spatiaux, à relever leur point nodal et les franges à partir desquelles elles se dissipent.

6 Par ailleurs, nous retiendrons quelques postulats, préambules à cette approche, dont le premier et le plus évident est que la ville ancienne existe, même si l'on ne peut être aussi affirmatif quant à ses limites. Elle ne peut être assimilée, à l'instar d'une formation socio-spatiale, à « un univers clos, parfaitement cloisonné. (...) Les formations socio-spatiales se définissent au contraire beaucoup plus par leur cœur que par leurs franges. De plus, elles ne structurent pas la totalité de l'espace et ne constituent pas une couverture géographique continue et hiérarchisée. » (Di Méo, 1991)

7 Pour reprendre les assertions de Robert Ilbert, la ville ancienne n'est « ni close, ni exclue, ni homogène » et « continue à jouer un rôle essentiel dans l'évolution globale de la cité ». Il serait aléatoire de vouloir la circonscrire et l'appréhender comme une entité fermée, isolée de l'ensemble urbain, comme une portion de ville confinée et figée ; au cœur de l'agglomération, elle est le lieu de transformations multiples s'exprimant dans le champ socio-spatial, tant dans les sphères publiques que privées. La ville ancienne ne peut être perçue comme le cadre déchu de la genèse de la cité et ne se réduit pas non plus à la fraction centrale la plus «populaire » de l'agglomération contemporaine.

8 En tenant pour acquis que «l'espace géographique est plus fait de superpositions et d'interférences que de limites $»^{4}$, la ville ancienne apparaît comme un sous-ensemble qui ne peut être décontextualisé. Elle ne peut donc être considérée comme un " conservatoire» des modes de vie et des pratiques, en marge de la société : «Les formations socio-spatiales n'enferment en aucun cas les idées, les pratiques et les attitudes des individus dans une détermination étroite. (...) Comme les structures, elles s'identifient à des systèmes de transformations, au sens où une modification de leurs composantes ne détruit pas forcément leur configuration d'ensemble. Dynamiques, elles intègrent le changement sans pour autant disparaître. Elles font preuve d'une permanence diachronique notable.» (Di Méo, 1991) «Les visages de la ville se 
brouillent », note André Raymond, «mais ce Caire en morceaux peut néanmoins être recomposé en ensembles plus ou moins cohérents, chacun révélant bien les profondes différences sociales qui le divisent. »

9 Nous utiliserons, pour construire notre présentation, cette notion de cohérence qui sous-tend également le concept des formations socio-spatiales : « unités géographiques cohérentes, espaces plus ou moins perceptibles et délimités, mais toujours suffisamment présents dans le sens commun pour faire l'objet de représentations collectives » (Di Méo, 1991).

Lire le passé et le présent sur les cartes

En réalité, ville fatimide, ville ayyoubide, ville mamelouke et ville ottomane (sans parler de la ville tulunide du XI ${ }^{e}$ siècle) se confondent; et si l'on parle aujourd'hui

de vieille ville, on fait en gros allusion à la cité du XVIII ${ }^{\mathrm{e}}$ siècle telle que nous la révèlent des plans comme celui de Niebhur (...) ou de la Description de l'Égypte. (llbert, 1982)

Le réflexe légitime de celui qui tente de cerner un espace est de vouloir l'englober d'un regard, identifier sa forme, le matérialiser sur les cartes. A regarder les cartes anciennes, l'on pourrait s'émerveiller de la permanence relative des tracés et être tenté de cheminer dans la vieille ville "en suivant " le plan de la Description. La ville à l'horizontale, en plan, semble remarquablement stable; associée aux voies percées ou aménagées aux $\mathrm{XIX}^{\mathrm{e}}$ et $\mathrm{XX}^{\mathrm{e}}$ siècles, la trame en multiples et délicates impasses, qui "authentifie" la ville "arabe-musulmane », est toujours là, certes parfois " décortiquée " : les Cairotes ont investi les espaces publics; des placettes ont disparu, de nombreuses impasses se sont transformées en ruelles, ou vice-versa. L'axe de la Qasaba $^{5}$, parcouru et maintenu par des monuments de diverses emprises, reste la voie centrale qui structure cet ensemble. Mais la «vraie» ville, celle en trois dimensions, sort totalement de ses empreintes, étalant sa complexité ; le remplacement rapide du bâti, sa "verticalisation ", les modifications du parcellaire, la projection à une autre échelle (celle de la mégapole) sont autant d'aspects qui altèrent la lisibilité cartographique et multiplient échelles, thèmes et cartes.

11 Le plan IGN de 1977 au 5.000e donne une vision de l'ensemble central mais homogénéise la ville en estompant les zuqâq, 'atfa et autres hâra $a^{6}$. Il ne rend compte ni de la plénitude, ni de la subtilité de la trame urbaine. Le millième, riche en toponymes et en indications diverses, est ancien : il a été réalisé entre 1909 et 1932. Le plan de Creswell, qui répertorie les monuments islamiques, est davantage un inventaire qu'une carte. Encore au millième, le plan parcellaire actualisé, réalisé entre 1982 et 1987 par l'Atelier du Caire ${ }^{7}$, permet une lecture fine de la trame urbaine et de l'occupation du sol. Il apporte encore une dimension supplémentaire en ce qui concerne la typologie du bâti, puisque l'essentiel des monuments (mosquées, madrasa, wakâla, sabîl, kuttâb, etc.) est représenté en plans coupés. Mais il est arrêté, dans sa partie occidentale, sur une ligne allant de la place 'Abdîn à la mosquée Ibn Tûlûn, frustrant la ville de son intégrité.

12 Le fond de la carte en annexe a été réalisé à partir du plan parcellaire muet de 1970 au 2.500e. Depuis sa publication, de nombreux changements sont intervenus dans le tissu urbain ; certains ont été identifiés, d'autres non. Il était impossible de les recenser tous, même en croisant les informations extraites des cartes précédemment citées; les modifications effectuées restent partielles, aussi ce plan recèle-t-il certainement des décalages diachroniques. Cette cartographie, image provisoire, est une étape d'un travail en cours, dont elle est le support, ce qui explique son aspect dépouillé ; elle n'a 
d'autre ambition que de présenter les formes de la ville ancienne dans leur simplicité première, l'îlot et la voirie. Elle se couvrira ultérieurement de noms, de signes, de trames et de sens.

Comment décider de ce qui est sur la carte, de ce qui en est exclu? Le repérage commence par l'identification de quelques percées, ou axes majeurs, auxquels pourrait être attribué un rôle de frontière, de limite. A l'est et au sud, de vastes cimetières sont là, qui séparent la ville ancienne d'autres lieux. Leur emprise peut même être imaginée, matérialisée en gris foncé sur une carte. Il y a aussi celui de Bâb al-Nasr, au nord, qui paraît à première vue superflu puisque situé au-delà des remparts. Mais ces murailles sont plutôt des supports aux constructions que des limites. L'ancien faubourg d'alHusayniyya, bien qu'extra-muros, ne peut être ignoré, il faut lui faire une place. De toute façon, il avoisine le cimetière de Bâb al-Nasr, qui n'est plus seulement une nécropole.

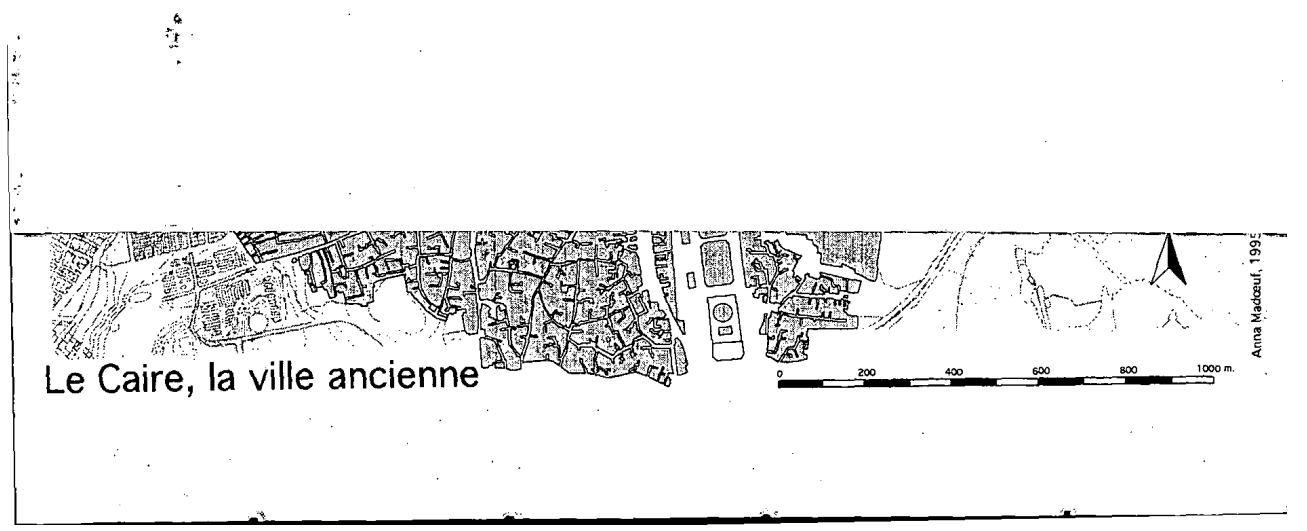

Il ne resterait plus qu'à « ranger » le centre-ville à gauche et la ville ancienne à droite, en se servant par exemple des places prévues à cet effet, 'Abdîn ou 'Ataba. Mais que faire alors de Sayyida Zaynab? Ainsi, on n'obtient que des points, des seuils dispersés, alors que l'idéal serait une ligne, ou au moins un semblant de tracé... Mais rien de tel n'apparaît dans le sillage méridional de la rue Port-Saïd. De plus, de part et d'autre, des groupes mal répartis semblent s'être trompés d'ensemble morphologique, de famille, comme si la ville, secouée, avait égaré des lambeaux ou dispersé des éléments tels que le quartier d'al-Ma'rûf, ou certains îlots au sud de la place Mustafa Kâmel, dont, note Sawsan Noweir, « le système de voirie et l'organisation parcellaire appartiennent plutôt aux configurations de la ville ancienne». A l'inverse, le quartier d'al-Hilmiyya, aménagé sur le Birkat at-Fîl, étang comblé au XIX siècle, tranche par sa voirie au quadrillage régulier; autour du quartier de Sayyida Zaynab coexistent plusieurs étapes et types d'urbanisation. Par ailleurs, le tracé des qism ${ }^{8}$, unités intermédiaires du découpage administratif, n'est pas non plus adéquat pour effectuer une partition.

Même si l'on sait que les formations socio-spatiales sont des formes dont les frontières - au contraire des centres, des réseaux et des structures - se reconnaissent difficilement, il faut, pour obtenir une carte, opérer des sélections. La mise en relief ou en lumière d'un espace rend souvent plats ou sombres ses alentours. Toutefois, pour que la déconnexion soit moins nette, on peut « tricher » en laissant apparaître, en sousimpression, la trame atténuée de la ville.

16 Il est des espaces récalcitrants qui s'accommodent mal d'être disséqués, qui plus est dans un format - celui dit «A3 » - orienté nord-sud avec un petit vide quelque part en 
bas pour la légende. Mais si la carte est « plus un portrait qu'un plan $»^{9}$, alors il faut en accepter le cadre.

Agréger et superposer l'espace

17 En élaborant une "anatomie " de la métropole du Caire basée sur des indicateurs déterminants d'un mode de vie - life style $e^{10}$ - à partir de données extraites des recensements de 1947 et de 1960, Janet Abu Lughod obtient une division de la ville en treize secteurs. L'un de ceux-ci, qu'elle définit comme "Medieval Cairo unreconstitued $»^{11}$, correspond presque de manière parfaite à la localisation de la ville aux périodes fatimide, ayyoubide et début mamelouke, soit "presque tout ce qui subsiste de l'héritage médiéval du Caire à son apogée ». Ce secteur est contigu à celui qu'elle appelle "Transitional Zone of Osmosis » (schématiquement, le centre-ville); les zones de contact entre ces deux ensembles sont soumises à une attractivité réciproque qui s'exerce sur leurs marges, où les indicateurs ont tendance à fusionner.

Trois observations peuvent être retenues des impressions "kaléidoscopiques" évoquées par Janet Abu Lughod : ce secteur est pauvre, incontestablement urbain, et sa vitalité se traduit dans la fusion des fonctions d'habitat, de commerce et d'activités, même si l'organisation de ces deux dernières repose sur des critères particuliers. "Le secteur a fait montre d'une capacité de survie remarquable: si mille ans d'histoire n'ont pas eu raison de lui, il trouvera sans doute les moyens de se perpétuer. »

Quinze ans plus tard, Galila El Kadi, s'intéressant à la division sociale de l'espace au Caire sur la base des statistiques du recensement de 1976, croise deux séries de paramètres : la proportion de population par qism de deux ensembles de CSP extrêmes ${ }^{12}$ et d'autres indicateurs tels que la densité, le degré d'équipement par qism, le taux d'illettrisme, etc. Ce croisement révèle une partition du Caire en trois espaces euxmêmes composés de sous-espaces; l'un d'eux regroupe les 5 qism de la ville "fatimide " avec le cimetière de Khalîfa ${ }^{13}$, ainsi que Bûlâq et Masr al-Qadîma. C'est l'espace où l'homogénéité sociale est la plus marquée. C'est là que résident, même si l'on y trouve aussi des membres des CSP supérieures, «le sous-prolétariat urbain, les chômeurs, les nouveaux migrants... tous les urbains pauvres à mobilité sociale bloquée. » Galila El Kadi compare dans ce contexte la ville ancienne aux zones d'urbanisation spontanée : «Plus on appartient aux strates inférieures (prolétariat et sous-prolétariat), plus on est concentré dans les secteurs centraux anciens et les zones périphériques de formation récente. » Autre point commun aux deux ensembles, même s'il n'a pas la même origine dans les deux cas : le sous-équipement, lequel s'est aggravé dans les quartiers anciens.

«Par une interpénétration pointilliste des caractéristiques propres à chacun de ces deux ensembles", la frontière entre ville ancienne et ville moderne s'est encore estompée. Galila El Kadi souligne que ce "nivellement apparent ", s'il n'est pas celui des différences sociales, permet cependant de réfuter la représentation courante de deux villes en opposition.

21 Une autre caractéristique que partagent tous les quartiers centraux est d'avoir vu, au cours des trois dernières décennies, décroître leur population au profit des zones périphériques. La mobilité résidentielle, de l'attraction de migrants à la répulsion de citadins, y a connu diverses phases en peu de temps. La dernière semble être marquée par des phénomènes de décohabitation, mais n'est pas encore bien appréhendée.

22 Si l'on ne peut plus parler d'un "espace-éponge » tel que celui décrit par Pierre Marthelot, les migrations vers Le Caire s'étant considérablement ralenties, rien ne permet non plus d'envisager une quelconque «désertion du centre» lorsque, à titre 
d'exemple, les densités des qism de Mûski et de Gamâliyya sont respectivement d'environ 1.100 et 800 habitants à l'hectare. Une autre façon de diviser la ville et d'agréger les espaces est celle des urbanistes, qui utilisent le concept de «secteur homogène " comme outil de la planification. La ville ancienne fait partie du secteur homogène central du Caire, lequel regroupe le Central Business District (centre-ville moderne), les zones intermédiaires et le Caire "historique »; mais paradoxalement, son assimilation à cet ensemble se fait au détriment de la multiplicité de ses fonctions et de sa centralité, puisque la place qui lui est dévolue dans ce cadre est celle de centre historico-touristique, et cette " vocation ", limitée, ne concerne que son centre ${ }^{14}$.

La ville en fonctions

Habiter, travailler et vivre sont des notions qui, dans la réalité, se mêlent, se complètent, se "répondent »; un des traits de la personnalité de la ville ancienne réside certainement dans leur complémentarité. Cet enchevêtrement des attitudes et des fonctions doit être souligné, puisque celles-ci ne seront, dans le cadre de cette analyse, abordées qu'isolément.

Du privé au public, du plus petit au plus grand, du logement où se love la vie familiale à la hâra, de la rue commerçante au parvis de la mosquée, de l'intime au collectif... A ces espaces fortement hiérarchisés correspondent autant de pratiques; si ces "principes » ne sont pas tous propres à la ville ancienne, la multiplicité des combinaisons qui en découlent lui est spécifique.

Georg Stauth relève l'opposition entre la rue, espace public, et la hâra, lieu de vie, espace privatisé, protégé, préservé : «L'espace, dans la hâra, est un espace libre pour ceux qui y vivent. » Vivre dans une même hâra représente plus qu'un simple voisinage : "L'habitus de la hâra est celui de la préservation de l'intimité. Rien ne doit être exposé, mais rien non plus ne doit être dissimulé. » NawaI al-Messiri Nadim montre comment la hâra peut être considérée comme une extension du logement, un haut lieu des sociabilités, tout particulièrement celles des jeunes et des femmes, et combien l'appartenance à la hâra est déterminante de l'identité, individuelle et collective. Les pratiques spatiales et sociales ne sont pas pour autant déterminées en fonction d'immuables critères sociaux, culturels et spatiaux. Comme l'ont montré Leïla Ammar et Mona Charara pour les hâra du Caire fatimide, le renouvellement du bâti modifie l'image et la fonction des espaces : des ateliers nouveaux s'installent, les quartiers sont ouverts aux « étrangers » qui viennent y vivre ou y travailler; toutes ces composantes contribuent à modifier les perceptions des quartiers. Les modes de vie évoluent, des tendances et des modèles nouveaux se font jour dans les pratiques quotidiennes, dans les domaines de la vie sociale comme au sein de la cellule familiale :

La hâra que décrit S. 'Uways n'a pas disparu. Et l'usage de la rue comme lieu d'une vie sociale ouverte, contrôlée par le voisinage, terrain de jeux et d'apprentissage urbain, continue d'être une pratique populaire qui déborde largement le cadre des quartiers anciens et des hâra proprement dites. Mais un autre modèle est venu s'y opposer : « vivre la porte fermée » et les enfants à la maison. (Depaule, 1990)

Par ailleurs, ce concept de hâra dont Jean-Charles Depaule a souligné la perméabilité, s'il est prégnant du point de vue identitaire, est producteur, en ce qui concerne les relations de voisinage, d'un discours attendu qui ne rend pas toujours compte des événements et des pratiques réelles de la vie quotidienne. Albert Camus, dans un autre contexte - celui des populations d'origine européenne à Alger dans les années $20-$ donne une illustration de ce décalage : 
Leurs familles étaient vaguement amies, ou comme on l'est dans ces quartiers, c'està-dire qu'on s'estimait sans presque jamais se rendre visite et qu'on était très décidé à s'aider les uns les autres sans presque jamais en avoir l'occasion. Seuls les enfants étaient devenus véritablement amis. ${ }^{15}$

Une des caractéristiques de la ville ancienne tient à une évidence : le fait qu'elle doive se renouveler dans un espace circonscrit; la dynamique de la construction y est vivace, tout particulièrement celle générée par sa population; le renouvellement du bâti courant est rapide (la majorité des édifices a moins d'un siècle) ; le tissu urbain est, de ce fait, extrêmement "vivant» et en métamorphose constante. Cette capacité de production, de modification, de remodelage et d'appropriation s'exerce également, à divers niveaux, sur l'espace public.

Cette dynamique est aussi le reflet de la vitalité du secteur économique : artisanat, petite industrie et commerce. La ville ancienne est le premier espace commercial de la ville. Bien sûr, les commerces de proximité y sont présents pour satisfaire les besoins d'un marché intérieur considérable, mais surtout, le commerce spécialisé y draine des flux de l'ensemble de l'agglomération.

Rien ne reste du grand commerce international qui faisait vivre le centre. (...) Pourtant, le site ancien du centre reste sans rival pour certains produits et certaines clientèles. Pour beaucoup de Cairotes et d'Égyptiens, au moins des classes populaires, l'axe Azbakiyya-Khân al-Khalîlî par la rue Mûski et la rue Azhar est un site phare du Caire, un repère fondamental. (David, à paraître)

La vieille ville a la quasi-exclusivité de l'artisanat destiné, entre autres, aux touristes, vendu sur place et diffusé sur l'ensemble du territoire égyptien. Elle est également spécialisée dans le commerce de la bijouterie et de l'orfèvrerie, du textile, des épices et du cuivre, de même que dans celui de nombreux et divers articles «rares » qui se trouvent moins aisément ailleurs. Que la fréquence de l'achat soit courante ou exceptionnelle, l'on se réfère à des quartiers ou des lieux précis pour l'acquisition d'une gamme de produits très étendue. Ainsi le textile, activité la plus importante et la plus dynamique du centre ancien, est aussi la plus concentrée avec environ 350 locaux regroupés autour de la partie médiane de la rue al-Azhar. Dans la partie occidentale de la ville ancienne sont rassemblés les produits "modernes-importés» de différente nature; il y a, près de la place 'Ataba, une rue pour les machines à coudre et leurs accessoires, une rue pour les cadenas, serrures, etc. Dans le même secteur se trouvent les montres, le petit électroménager, la hi-fi ou la vidéo.

La partie nord de la rue al-Mu'izz, après le secteur d'al-Sâgha - les orfèvres - propose des tables, des dominos, des pipes à eau, autant d'équipements pour les cafés. Rue Ahmad Mâhir, près de Bâb Zuwayla, se concentrent des graineteries, des marchands de parasols et de chaises pliantes, des boutiques d'accessoires de jardinage et des marbriers; pendant le mois de Ramadan, c'est aussi là que sont vendues les lampes fabriquées à cette occasion. Hormis ce commerce temporaire, les autres produits sont essentiellement destinés à une clientèle extérieure à la ville ancienne.

31 La rue du Mûski, une des rues commerçantes les plus fréquentées et les plus animées du Caire, est l'espace du prêt-à-porter, de ses accessoires et du textile. Jean-Claude David relève le dynamisme et la particularité de cet axe :

«C'est un souk généraliste sur le passage obligé vers les souks spécialisés; il profite de leur attractivité et réciproquement.» On peut ainsi, rue du Mûski, s'équiper entièrement pour un mariage, que ce soit pour l'achat de la robe de mariée, des lustres, 
de la vaisselle, des cotillons ou des dragées (produits fabriqués sur place), et poursuivre jusqu'aux bijoutiers d'al-Sâgha.

33 La ville ancienne est plurielle et les espaces qu'elle abrite, selon leurs caractéristiques et leur localisation, s'avèrent des supports très divers aux mécanismes de dynamiques comme la construction ou l'implantation des activités, mais des processus communs se font jour sur l'ensemble de ses territoires. C'est ce qui ressort d'une enquête réalisée en 1990 dans trois secteurs distincts : le Mûski, contigu au centre-ville, Hârat al-Yahûd au centre, et l'extrémité orientale de Gamâliyya ${ }^{16}$.

Dans les deux premiers quartiers, centraux, la spéculation est intense; chantiers et immeubles récents occupent une part importante de l'espace urbain et les terrains vacants sont presque inexistants, de même que les constructions basses. Par contre, à Gamâliyya, terrains vagues, bâti hétéroclite et immeubles en ruines marquent le paysage urbain. Cependant, sur tous ces espaces, la pression de l'artisanat, de la petite industrie et du commerce transforme chaque parcelle libre et surtout constructible - à Gamâliyya, les terrains waqf, souvent gelés, sont nombreux - en un nouvel immeuble destiné à ces activités.

D'un petit bâtiment consacré à l'artisanat, enclavé sur une parcelle étroite à Gamâliyya, à un «immeuble industriel» d'une centaine d'ateliers orienté sur deux rues et construit à l'emplacement d'une synagogue, le dynamisme de la construction de ce type d'immeubles témoigne de la vitalité économique de la vieille ville et des mutations qui en découlent. On observe la multiplication, la diversification et la modernisation de certaines entreprises, mais elles sont toujours tributaires de leur sectorisation. L'éventail des possibilités est souvent large, mais il dépend d'un " thème » dominant ; lorsque des ateliers apparemment indépendants se côtoient, ils sont souvent liés à la même chaîne de fabrication. Cette typologie est ancrée dans l'espace : en général, les ateliers liés à une chaîne de fabrication sont mitoyens. Cet état de fait n'est pas synonyme d'inadaptation et ne contrarie pas la fabrication de produits d'apparition récente. Cette organisation perdure au sein des nouveaux immeubles d'activités et dans divers secteurs économiques.

A titre d'exemple, à Gamâliyya, tout un étage fabrique des plateaux en cuivre: du polissage à la décoration peinte, toutes les étapes du travail sont présentes, concentrées non plus à l'échelle d'une rue mais à l'intérieur d'un même bâtiment. A Hârat al-Yahûd, de gros immeubles concentrent la production de toute une gamme de vêtements, chaussures et accessoires; tout est réalisé sur place, du tissage aux finitions. Cette sectorisation est bien sûr plus ou moins marquée selon la typologie de la localisation et selon la dichotomie commerce-production. Le long des axes importants et près des zones de passage, il y de nombreux magasins et le commerce est différencié. Au cœur de l'îlot, par contre, les ateliers sont majoritaires et leur logique de distribution plus évidente. Enfin, chaque quartier est lié à un (ou plusieurs) pôle(s) d'attraction qui sont autant de lieux d'écoulement des marchandises. Gamâliyya dépend du Khan al-Khalîli, le Mûski est essentiellement en relation avec le centre-ville ; quant à Hârat al-Yahûd, il fournit les commerces de la rue du Mûski consacrés à l'habillement. Cependant, les nouveaux immeubles d'activités s'implantent de plus en plus loin de ces pôles économiques; on trouve des bâtiments destinés à la confection jusqu'à la limite nord de Hârat al-Yahûd, et à l'artisanat jusqu'à l'extrémité orientale de Gamâliyya. autant. Ainsi, une part de la production de Gamâliyya est exportée vers diverses villes 
d'Égypte, mais transite auparavant par le Khan al-Khalîli, dont les commerçants sont aussi des intermédiaires. Aussi les livraisons deviennent-elles plus volumineuses mais moins fréquentes. Cet état de fait contribue au développement de la fonction de stockage, désormais présente dans chaque immeuble d'activités, et qui peut, dans les cas extrêmes, occuper tout un bâtiment - d'où l'apparition, surtout à proximité du centre-ville, « d'immeubles-vitrines » regorgeant de marchandises.

Certaines branches du secteur du tertiaire supérieur (services divers), jusque-là inexistantes, apparaissent sur les franges au contact du centre-ville, et le long de la rue al-Azhar (banques).

Lors de l'enquête, à de rares exceptions près, tous les chantiers, même localisés dans des impasses ou sur des voies d'accès peu commodes, étaient ceux d'immeubles destinés à l'artisanat, au commerce ou au stockage (ou à un panachage de ces trois fonctions).

41 Fortement dépendante de la morphologie urbaine, l'organisation fonctionnelle de l'espace reste encore marquée par la séparation des zones vouées à l'habitat, aux activités de production et au commerce, mais ces deux dernières fonctions débordent largement hors de leurs sites d'implantation originelle et investissent des lieux d'où elles étaient auparavant absentes. Ce phénomène de pénétration des fonds d'impasses et des hâra traditionnellement réservés à l'habitat modifie la hiérarchie fonctionnelle préexistante. On assiste à un redéploiement de la distribution des fonctions, la sectorisation s'estompe tandis que l'imbrication se diffuse à des échelles diverses puisqu'au sein d'immeubles anciens, des logements peuvent être transformés en ateliers.

D'autres études sur les quartiers centraux de la ville ancienne ont montré, par ailleurs, les capacités de rapide renouvellement des secteurs de l'artisanat et de la petite industrie. Ainsi Günter Meyer, sur la base d'une enquête menée en 1986 sur les entreprises artisanales de Bâb al-Sha'riyya et de Gamâliyya, notait que les deux tiers de celles-ci avaient été créées depuis 1980 et insistait sur leur adaptabilité en montrant comment le secteur de l'aluminium, en crise, se reconvertit dans le travail du cuivre. Mais selon l'optique d'André Raymond, les conséquences de cette évolution, du point de vue des nuisances diverses et de la dégradation du bâti qui en résulte, peuvent s'avérer négatives :

Un des traits les plus marquants de l'évolution récente du centre ancien est le développement des ateliers de petite industrie productrice de biens de consommation courante apparus à partir du début des années 80 , principalement grâce aux revenus de l'émigration. Une revitalisation des quartiers anciens sous cette forme industrielle peut signifier à terme un arrêt de mort.

Rythmes et symboles, centralité et sacralités : le temps des pèlerinages

André Raymond énonce ce qui pourrait être fatal à la ville ancienne; Jacques Berque, en conclusion de son analyse de la Gamâliyya, s'interroge en revanche sur le phénomène inverse : "Qui sait si la puissance du cadre monumental, son chargement d'histoire, sa qualification rituelle, ne sont pas ce qui a permis jusqu'ici au système de se survivre dans une substitution quasi totale de ses contenus humains?"

La ville ancienne est incontestablement un espace riche en formes et paysages urbains, et présente une grande diversité de types architecturaux. Ce patrimoine, omniprésent, façonne la représentation de l'espace; les monuments qui le ponctuent, caractérisés par leur multitude et leur diversité, sont autant de signes, de repères et de référents 
identitaires. « Au Caire, le passé existe encore souvent à l'état brut. Selon les quartiers, il est encore tel quel, quotidien, fonctionnel et non décoratif - il commande encore une partie de l'espace urbain; il est présent, visible; parfois incongru, souvent scandaleux. » (Bénard, 1991)

La ville ancienne est le lieu privilégié de l'expression des pratiques religieuses collectives, comme les mawlid ${ }^{17}$ qui, à travers le culte d'un saint, mettent en scène un quartier selon un rituel où la pratique codifiée de l'espace joue un rôle majeur. Le mawlid est aussi une interface : entre Le Caire et la province, entre le quartier et la ville, entre la baraka et le pèlerin, entre le sacré et le profane. Le mawlid est une célébration religieuse qui inclut des éléments de fête foraine.

Les principaux mawlid, ceux d'al-Husayn, de Sayyida Zaynab et du Prophète, ont accueilli chacun environ un million de personnes en $1994^{18}$. Si les Cairotes s'y rendent nombreux, les pèlerins viennent de toute l'Égypte ${ }^{19}$, essentiellement sous la bannière des diverses confréries soufies, et nombre d'entre eux restent pendant la dernière semaine de la fête. Des chapiteaux à motifs géométriques colorés s'élèvent un peu partout, surmontés de banderoles qui rappellent, outre l'affiliation confrérique, l'origine géographique des pèlerins, la représentation temporaire, au cœur de la ville, des provinces : " Tous les Saints de Louxor ", « Qâdiriyya de Mansûra », etc. Les mawlid sont des événements cycliques qu'accompagnent des pratiques exceptionnelles. Ainsi le temps public, celui des festivités, implique l'espace public en lui donnant son intensité maximale. Ces rythmes « exacerbent » les espaces publics et atténuent aussi la privatisation des lieux investis par les pèlerins, installés dans les tentes disséminées aux alentours des mosquées et jusque dans les impasses. Les mawlid importants sont en partie supervisés par les autorités religieuses et civiles et les confréries soufies, mais les très nombreux petits mawlid « de quartier $»^{20}$, qui ponctuent l'espace et le temps, sont organisés par les habitants et s'avèrent souvent les lieux de pratiques moins orthodoxes. Ces manifestations, espaces de la transgression d'interdits sous couvert de célébration religieuse, pourraient être assimilées à des " antimondes ", des espaces de « dérogation ou de dérégulation $»^{21}$.

Peut-être est-ce parce qu'elle semble tellement évidente que la fonction religieuse et culturelle de la ville n'est pas évoquée à sa mesure. «Interrogés sur le silence de la presse à propos de l'affluence record au mawlid d'al-Husayn, de nombreux Égyptiens firent cette réponse : «On n'en parle pas parce que c'est normal. Les médias vont-ils prendre la peine de mentionner le lever ou le coucher du soleil ? » » (Luizard, 1990)

Contrairement à la plupart des événements, les mawlid se "bonifient » avec le temps : ainsi a-t-on dit, de ceux d'al-Husayn et de Sayyida Zaynab, que "c'était mieux cette année ». A Sayyida Zaynab, la différence était manifeste en raison de l'abondance des illuminations, ceci grâce au député du quartier et président de l'Assemblée du Peuple, M. Fathi Surûr. Ce dernier - candidat probable aux élections législatives de l'automne 1995 - était d'ailleurs célébré par des portraits et des banderoles à son nom.

50 Les grands mawlid sont aussi des espaces de représentation à des niveaux très divers. La foule est composite : on reconnaît les pèlerins provinciaux, Sa'îdi (habitants du sud de l'Égypte) et autres, à leurs vêtements. Les familles tentent d'éviter la cohue ; les jeunes des quartiers proches s'y précipitent, les garçons en bande, les jeunes filles en petits groupes. Les enfants se pressent autour des attractions foraines, hésitent entre la roulotte des tortues Ninja et celle d'Abû Zayd. La police, en civil ou en uniforme, est présente. Et sous les chapiteaux qui ont le privilège d'être installés sur la place même, 
le Conseil soufi et les confréries les plus dynamiques, celles dont les shaykh sont les plus charismatiques ou influents, se donnent à voir. Plus on s'éloigne de la place et plus les bruits de fête - carabines, pétards, stands de forains - recouvrent celui de la célébration, échappé des tentes où l'on récite le Coran et où l'on pratique le $d h i k r^{22}$. "Tout le monde ramène des pois chiches du mawlid ", dit le proverbe... L'éventail de ce que chacun vient y chercher est éclectique: animation, jeux, rencontres, prières, baraka, transe, ou un peu de tout cela.

51 Facette primordiale de sa personnalité, la « sacralisation » de la vieille ville, où chaque quartier est placé sous l'aura d'un saint, est déterminante de la perception qu'en ont tant ses habitants que les autres Cairotes. Ainsi l'espace cognitif de Hamed, personnage d'une nouvelle de Youssef Idris, se construit autant autour du quartier où il réside que par rapport aux deux grandes mosquées : «Il a appris à se mouvoir dans la ville : il sait se rendre jusqu'à la mosquée de Sayyida Zaynab, il peut traverser de part en part tout le quartier de Choubra, et connaît précisément l'itinéraire pour atteindre le mausolée d'el-Hussein.»

Si nous récapitulons tous ces lieux rituels, toutes ces mosquées, tous ces oratoires, toutes ces zâwiya, tous ces mausolées de saints, tous ces lieux de réunion mystique, d'observance ou de pure et simple surérogation - pour ne pas dire superstition, car il s'agit en bien des cas d'un islam populaire fort éloigné de l'orthodoxie -, nous constatons à quel point la sacralité afflue et s'ordonne selon une structure liturgique qui épouse étroitement la figure et l'économie de la ville ancienne. (Berque, 1969)

Les espaces centraux de la ville ancienne sont à la fois lieux de tourisme, lieux d'affluence des provinciaux durant les mawlid et lieux de «communion» des Cairotes qui viennent, rarement mais rituellement, toutes classes sociales confondues, participer massivement aux veillées de Ramadan à al-Husayn, goûter une spécialité culinaire populaire, une boisson, ou seulement l'animation. Cette apparente flânerie prend la forme d'un pèlerinage vers des espaces symboliques qui représentent la facette baladi ${ }^{23}$ de l'identité égyptienne, ou au moins cairote. La ville ancienne est ainsi liée aux circonstances qui rappellent ou appellent la tradition; mawlid, Ramadan... Cette figuration de la ville ancienne fait de sa représentation un espace consensuel, celui des valeurs culturelles traditionnelles, autour desquelles la société tout entière peut se reconnaître et se retrouver.

Les éléments qui symbolisent la vieille ville peuvent être reproduits dans d'autres lieux. Ainsi cette année, durant le mois de Ramadan, plusieurs grands hôtels avaient aménagé des espaces baptisés, en général, de noms d'oeuvres de Naguib Mahfouz $^{24}$, où étaient constituées en décors de référence des ruelles imaginaires de la ville ancienne, avec minarets et moucharabiehs, accessoires et figurants: serveurs en galabiyya, serveuses en robes imprimées et melâya, charrettes de vendeurs ambulants, boutique d'artisanat, café oriental et restaurant où l'on pouvait consommer, de l'iftar au suhûr, boissons et plats populaires du Ramadan, dans un cadre et une ambiance voulus baladi...

Les rythmes de la ville ancienne sont en décalage avec ceux du reste de la ville, les horaires de bureau n'y ont pas de sens, les embouteillages ne s'y produisent pas aux mêmes heures, le vendredi y est jour d'activité. Cette temporalité varie aussi en fonction notamment des heures, des jours et des saisons, lesquelles ne s'y manifestent pas seulement dans les vitrines des boutiques de vêtements, mais influent sur l'organisation de la vie sociale et familiale. 

l'élaboration, sans cesse remodelée, d'une citadinité particulière. La fonction religieuse et culturelle est prégnante et indissociable de la fonction d'ouverture, de la capacité d'intégration et de la cohésion de la ville ancienne, espace pluriel caractérisé par la diversité de ses quartiers, des types de lieux qu'ils abritent et des pratiques qu'ils engendrent. Sensible également aux temporalités, la pratique des espaces est tributaire de ces variations qui mettent en jeu un ballet d'acteurs - endogènes ou non - prenant tour à tour place sur un territoire dont l'usage se redéfinit.

La Gamâliyya encore et toujours

56 Les contours d'un espace s'avèrent souvent fluctuants, difficiles à préciser, mais le centre est presque toujours identifié, matérialisé et symbolisé ; ainsi de l'univers à la terre... et de la ville ancienne, voire du Caire, à Gamâliyya, à la croisée de l'axe millénaire de la Qasaba et des percées du XIXe et du XXe siècles, les rues du Mûski et d'al-Azhar.

Les franges et les marges sont par définition floues, mouvantes et parfois malléables, toutes les superpositions ne coïncident pas, mais malgré l'utilisation de grilles d'analyse différentes dans l'appréhension de la vieille ville, toutes s'ajustent sur un centre, Gamâliyya, qui apparaît comme cœur et point nodal.

Qu'un dessin tente de représenter "l'image arrêtée d'une portion de rue », comme pour confirmer la "signification obstinée » du quartier exprimée par Jacques Berque, et la rue choisie est celle de Gamâliyya, seule rivale de la Qasaba. «Pour beaucoup d'Égyptiens, Gamâliyya est l'Égypte ", note Georg Stauth en introduction à son étude sur ce quartier. La Gamâliyya, "écoumène » ou vedette, n'a-t-elle pas obtenu, par l'intermédiaire de la littérature, le prix Nobel?

Dans la Trilogie de Naguib Mahfouz, Gamâliyya, al-Husayn et le Khan al-Khalîli apparaissent, pour ceux qui y vivent, comme des quartiers différenciés. Mais l'espace s'est redéployé, les quartiers brouillés, le visage de Gamâliyya s'est troublé mais enrichi puisque se dégage, dans les perceptions, une assimilation de ces secteurs, tant pour les habitants de la vieille ville que pour les autres Cairotes. Le Khan al-Khalîli, les grandes mosquées d'al-Azhar et d'al-Husayn, les monuments et le prestige de Gamâliyya, éléments attractifs sur une zone d'influence commune et de proximité, ont permis, malgré (ou grâce à ?) leurs fonctions antithétiques, une transcendance et une synthèse spatiales par la représentation et la cohésion d'un centre de référence.

Quartier populaire (sha'bi), «appauvri mais non déchu " $^{25}$, il bénéficie d'un statut particulier, celui de wast al-wast ${ }^{26}$. «Les gens viennent ici de tous les coins du monde »; "Quand je voyage à l'étranger et qu'on me demande d'où je viens, je réponds: de Sayyidna al-Husayn!» affirment respectivement Hagga Sayyida et Osta Khamis ${ }^{27}$. La relation au centre, totale, $s^{\prime}$ exprime de manière centrifuge et centripète.

61 La mosquée d'al-Husayn, sanctuaire affectivement le plus important et le plus visité d'Égypte, est «le véritable centre sacré du Caire, le point de ralliement de toutes les cérémonies religieuses » (Luizard, 1990).

62

Quant à l'institution millénaire d'al-Azhar, elle est le siège d'une université prestigieuse qui accueille des étudiants dont beaucoup viennent d'autres pays musulmans ${ }^{28}$ et, surtout, elle propage une voix symbolique et spatialisée ("al-Azhar a dit»), qui se prononce sur des domaines aussi divers que l'excision ou le dernier film de Youssef Chahine, et dont l'influence se diffuse dans toute l'Égypte.

Égypte/Monde arabe, 22 | 1995 
63 Les environs des grandes mosquées et le Khan al-Khalîli sont des espaces d'activités nobles, l'apprentissage y est réputé. Le Khan al-Khalîli est le souk de référence en Égypte : il rassemble environ un millier de boutiques dont certaines ont des annexes dans les grands hôtels, les quartiers chics ou d'autres villes d'Égypte ; il est un lieu de passage obligé pour les touristes, dont les circuits sont en général limités à ses environs. Entre al-Azhar et les quartiers de Bâb al-Nasr et Bâb al-Futûh, concentrés essentiellement sur les rues al-Mu'izz et Gamâliyya, plus d'une centaine de monuments, soit les deux cinquièmes du bâti, témoignent des époques fatimide, ayyoubide, mamelouke, ottomane ainsi que du XIx $x^{e}$ siècle.

Dans ce cadre, Gamâliyya est le seul quartier de la ville ancienne à être projeté dans l'avenir par "une opération ambitieuse de réhabilitation urbaine", le projet NordGamaliyya ${ }^{29}$, lequel doit assigner au quartier un rôle dans la ville " actuelle moderne ", celui de centre historico-touristique nécessaire mais circonscrit. Le slogan du projet, " une porte ouverte sur le fabuleux trésor des monuments islamiques ", fait référence à un quartier touristique idéal basé sur la réhabilitation et la mise en scène des remparts et des portes du nord de la ville ancienne, la création d'hôtels, de centres commerciaux et l'aménagement d'un circuit balisé. L'un des quatre éléments d'un "scénario de l'inacceptable » imaginé par les urbanistes pour Le Caire du xxle siècle est la destruction du patrimoine urbain. Le monument devient, dans ce contexte, le prisme à travers lequel sont révélées les carences affectant la ville ancienne : il faut rénover les égouts dont les fuites endommagent les fondations, la voirie doit être améliorée pour la mise en valeur du patrimoine architectural, les constructions adossées aux remparts doivent être détruites car elles les dissimulent, etc. Les habitants, quant à eux, apparaissent comme des « prédateurs » de monuments.

Gamâliyya, dans la description de la mégapole de l'an 2000, sera, plus que jamais, fatimide: «Les quartiers historiques de la ville fatimide ont développé les vocations artisanale, commerciale et touristique qui font leur originalité. $»^{30}$

La vieille ville et la grande ville, permanence et perméabilité

La ville ancienne est un ensemble caractérisé par un tissu morphologique et un tissu social particuliers, et surtout par l'interférence manifeste de leur superposition. Sur cet espace, l'imbrication des fonctions d'habitat, de commerce et de production (artisanat et petite industrie) s'intensifie et se généralise, même si, dans le détail, elles peuvent s'exclure.

67 Autrefois unique, cet espace a perdu depuis plus d'un siècle le «monopole de ville » et par conséquent de centre, qu'il partage désormais avec d'autres secteurs de la cité. Dépositaire et détentrice des fonctions «traditionnelles ", la vieille ville est perméable aux nouvelles donnes de la centralité, celle-ci se reformulant au fur et à mesure de l'extension et de l'évolution de l'agglomération.

Les dynamiques urbaines y sont d'autant plus remarquables qu'elles s'inscrivent dans un tissu caractérisé paradoxalement par la pérennité des tracés et par le rapide renouvellement du cadre bâti. Les contraintes morphologiques inhérentes à l'histoire urbaine peuvent, alliées à la dynamique du centre, se caractériser par une inventivité traduite entre autres par la réadaptation et la réutilisation de modèles urbains fonctionnels, comme celui de la wakâla réexprimé en partie dans les bâtiments neufs destinés aux activités artisanales et commerciales. Le cadre «mégalopolitain » du Caire 
est déterminant de la vitalité de la ville ancienne ; la mise en relation des flux qui en découlent renforce ses polarités.

Paradoxalement, le paysage socio-spatial se perpétue et se recompose dans le visible et l'invisible, dans le réel et l'imaginaire. Des indices de mutations s'ébauchent, certaines pratiques sociales sont affectées, d'autres conservent leur acuité; toutes, liées à une homogénéité sociale marquée et aux typologies spatiales, sont cependant réceptives aux variations des modèles comportementaux. Tous ces critères de cohésion confèrent une unité et une personnalité à la ville ancienne par rapport aux autres ensembles cairotes. Elle est de surcroît, pour ceux qui y vivent mais aussi pour les autres, l'espace de l'histoire et du sacré.

Mais quels sont le sens, les perceptions et les représentations qui peuvent émaner de cet espace dans le contexte contemporain du Caire? Comment la ville ancienne s'identifie, se scinde ou s'agglomère-t-elle? Quelle est sa composition dans la ville actuelle? Le Caire, en arrière-plan du roman de Sonallah Ibrahim, Les années de Zeth, semble privé d'espaces différenciés ; la ville est uniforme mais déroutante, les individus se retranchent dans la sphère domestique, lieu de l'investissement spatial, affectif, financier. «A l'ère de la mégapole, la ville n'est plus spatiale. Elle est médiatique. Elle est un tissu de flux, un tissu d'informations, un tissu de sens. $»^{31}$

71 La mégapole est difficile; c'est en partie cette difficulté qui transcende et généralise les espaces; c'est elle aussi qui, de manière maligne, unifie et réconcilie la ville. Cette perception se précise dans Epître des destinées, de Gamal Ghitany, où la ville ancienne n'est plus qu'une somme de lieux et en connaît le lot commun; de multiples signes, à peine perceptibles, y diffusent, comme ailleurs, une sensation de trouble. Auparavant, dans la vieille ville, l'espace primait et déterminait le temps, lequel n'y avait pas le même sens qu'ailleurs. Cependant, les "années funestes » ne vont pas l'épargner et corrompront même, dans le périmètre sacré de la mosquée al-Husayn, le gardien du mausolée de Qalaoun, qui vit dans une " portion de la rue el-Muiz, bloc compact extrait d'un temps révolu; et tandis qu'Achour veille sur elle, elle semble lutter contre l'usure, tendre vers l'éternité.» Mais le gardien, pour des raisons peu explicites, va se détourner de sa tâche ; la symbiose entre habitants et espaces se défait. Comme Achour délaisse le mausolée, l'aura de la mosquée al-Husayn devient inopérante. "Tant qu'ils restaient dans les parages, ils étaient à l'abri de ce qui les menaçait »... Mais la magie n'est plus, chaque être est happé par sa destinée, désormais détachée de celle des lieux. Le devenir de ces derniers reste aléatoire.

72 De même, le médecin dévoué qui vit entre centre-ville et vieille ville, rue al-Gaych, n'est plus le même à la suite de la démolition d'un immeuble ancien près de chez lui. Désormais il se passionne pour les terrains vagues, lesquels, en ville et surtout dans ce secteur, peuvent devenir les terres promises de la spéculation foncière. Quant au jeune calligraphe, son détachement sera absolu puisqu'il est contraint de partir à l'étranger : la ville ancienne, autrefois espace attirant pour les provinciaux, est devenue stérile et répulsive pour les siens. Tous les symptômes et symboles d'une mue sont exprimés, chaque personnage trahit les valeurs qui lui étaient chères et se distancie ainsi de son quartier, de son identité.

73 La ville ancienne n'est plus le bastion décrit par Naguib Mahfouz. Elle n'incarne plus le «fond de la ville ${ }^{32}$; d'autres lieux, forcément plus excentriques, l'ont remplacée dans ce rôle d'extrémité spatiale et sociale. Lieu de mémoire "sans histoire ", la contestation et la rébellion, sous leur forme contemporaine, semblent l'avoir désertée au profit des 
périphéries.. Depuis les opérations de lutte contre les stupéfiants menées par les autorités dans le quartier de Batniyya ${ }^{33}$, investi plusieurs fois par la police et l'armée entre 1974 et 1981, et hormis un affrontement à Sayyida Zaynab, en février 1994, entre la police et des islamistes, on évoque davantage, quand on parle de la ville ancienne dans les médias, ses monuments qui se fissurent et ses immeubles qui s'écroulent que sa société. Le tremblement de terre de 1992 a encore accentué cette représentation de la ville ancienne comme espace d'où émerge une cohorte de monuments fragiles et délabrés, au milieu d'activités et d'humains dont le trop-plein pourrait être transplanté vers les espaces périphériques vides que sont les new-settlements.

Les limites vacillent, le sens et le statut des espaces évoluent, quelle que soit leur position. Les grandes places ne sont plus des frontières, symboles de transition, les quartiers se mêlent; ainsi, la rue du Mûski, qui traverse incontestablement la ville ancienne, est extra-territorialisée dans sa représentation puisqu'on la désigne dans son usage comme appartenant à wast al-balad. De même, le temps est révolu où l'on pouvait vivre dans des espaces retranchés et étanches: la ville, en s'agrandissant s'est décloisonnée. «Le Caire est une mégapole ouverte et fragmentée» (Depaule, 1991) mais ces fragments sont diversement réceptifs à l'ouverture. Et celle-ci se propage inégalement selon les sphères, imprègne de manière différenciée les strates spatiales et sociales. L'organisation et les repères fondamentaux de l'espace et de ses usages perdurent, l'anomie n'est que partielle.

76 «Au café, un inconnu. Dans notre ruelle, les inconnus captent tous les regards; chacun se demande d'où vient cet homme. » Le sens qui émane de ces phrases, relevées dans Récits de notre quartier de Naguib Mahfouz ${ }^{34}$, se retrouve, inaltéré, dans celles de Gamal Ghitany à propos de la rue Husayniyya : « II en aime le brouhaha chaleureux et ne peut l'observer qu'en passant : il ne connaît personne dans le coin. S'il s'installe à la terrasse d'un de ces petits cafés, tout le monde va le regarder. L'endroit, quoiqu'à sa portée, lui est étranger.»

De même, résider dans la ville ancienne signifie aussi vivre dans le halo d'une mosquée, être dans l'aura d'un lieu saint; dans une nouvelle de Yahia Haqqi, La lampe de Oum Hachem, Ismaël vit à Sayyida Zaynab, «à l'ombre tutélaire de la Sainte ; ses fêtes et ses « saisons » étaient les nôtres, et le muezzin de sa mosquée nous servait de montre ». Les personnages d'Epître des destinées ont conservé ces mêmes repères et vivent « dans une vieille maison, au coin d'une ruelle où l'on apercevait la mosquée Ibn Touloun » ou « dans une maison de deux étages située près de Oalaat al-Gabal, et d'où l'on pouvait voir, pourvu que l'on se tînt à l'entrée de la ruelle, le minaret de la mosquée Mohamed Ali».

78 «L'Ezbékieh n'est pas loin de la Gamâliya dans l'espace, non plus que dans les mœurs. » Cette double proximité, relevée par Jacques Berque, s'est encore accentuée ; les deux centres sont devenus mitoyens. On peut désormais «voler» de l'un à l'autre sans traverser d'espace intermédiaire, en empruntant Ie fly-over, pendant aérien de la rue alAzhar, lequel décolle de l'Azbakiyya, surplombe la place 'Ataba, frôle les étages supérieurs ou les toits-terrasses encombrés des immeubles de la vieille ville et atterrit devant le complexe monumental du sultan al-Ghûrî. Jusqu'à une époque récente, on pouvait même bondir d'un Benetton à l'autre ${ }^{35}$. obsolète. Aucune ne correspond pleinement et exclusivement à ces qualificatifs, et 
toutes deux sont centre; à une autre échelle, la nouvelle opposition se situe entre la ville qui serait «spontanée » et celle qui ne le serait pas. Mais la ville ancienne peut, elle aussi, se révéler « spontanée ».

«Transformations, densifications, surélévations et constructions récentes nous apportent la preuve des potentialités de renouvellement du bâti liées à la quasi-absence d'un contrôle administratif et à l'initiative des habitants de la vieille ville. » (P. Panerai, 1991) Dans le même sens, Galila El Kadi note une "prolifération des constructions sauvages de type «taudis» dans les espaces vides à la place des ruines». A treize kilomètres de là, André Raymond retrouve, à al-Marg, dans une zone d'urbanisation spontanée, "des petits commerces d'alimentation et des marchands ambulants qui rappellent ceux de la vieille ville et des villages de la campagne». Il ne s'agit pas d'échapper à une opposition au profit d'une assimilation, mais d'évoquer les "catégories de l'urbain» (dont "vieille ville» fait partie), lesquelles sont souvent exclusives; en ce sens, l'intérêt de la méthode des formations socio-spatiales réside surtout dans la contextualisation de son appréhension de la complexité et de la globalité.

81 Ces rapprochements resteront donc les seuls, la centralité et l'intégration étant, parmi d'autres, deux attributs de la ville ancienne qui empêcheraient de poursuivre un tel raisonnement. Ainsi, lorsque pour une partie des Cairotes la vieille ville incarnait le "fond de la ville ", cette perception n'était peut-être pas partagée par ses habitants : «Moi qui vivais en plein milieu de la ville, dans le quartier de Sayyida 'Aysha, rue alZarâyib... », raconte Sayyid 'Uways. Aujourd'hui, les habitants des périphéries, nouveau " fond de la ville », peuvent difficilement se percevoir ainsi.

Lieu d'animation, d'échange, de rencontre, territoire du festif, des célébrations, du sacré, de l'histoire, du consensus; centre aux référents multiples, la ville ancienne existe autant par ses réalités que par ses représentations, diversement façonnées par cet ensemble de valeurs ; en cela, elle s'exprime en espace-enjeu. L'originalité sociale de la ville ancienne tend à s'atténuer, certains de ses particularismes morphologiques s'estompent. Parallèlement, ses fonctions symboliques se maintiennent, se renforcent même. Elle exprime une remarquable réalité idéelle; elle est à l'unisson avec la cité, centre-ville et vieille ville composent un rythme central à deux temps.

\section{BIBLIOGRAPHIE}

Abu-Lughod J., Cairo 1001 Years of the Cily Victorious, Princeton Univ. Press, New Jersey, 1971.

Al-Messiri Nadim N., « The Concept of the Hâra. A Historical and Sociological Study of AISukkariyya ", Annales Islamologiques, tome XV, IFAO, Le Caire, 1979.

Ammar L., Charara M., «A travers les hâra du Caire fatimide », Égypte/Monde arabe n 5, 1er trim. 1991, Cedej.

Baker F., « Questions de toponymie », Égypte/Monde arabe $\mathrm{n}^{\circ}$ 5,1 $1^{\mathrm{er}}$ trim.1991, Cedej. 
Battain T., Labib A., « Le Caire-mégalopole perçue par ses habitants », Égypte/Monde arabe $\mathrm{n}^{\circ} 5,1^{\mathrm{er}}$ trim. 1991, Cedej.

Berque J., Al-Shakaa M., « La Gamâliya depuis un siècle », Colloque international sur l'histoire du Caire organisé par le Ministère égyptien de la Culture au Caire du 27 mars au 5 avril 1969, RDA, 1972.

Benard M.-C., «Impression et surimpression urbaines », Égypte/Monde arabe n 5, $1^{\mathrm{er}}$ trim. 1991, Cedej.

David J.-C., "Centralités anciennes et actuelles dans Al Qâhira », à paraître dans Établissements de rapport au Caire aux époques mamelouke, ottomane et contemporaine, IFAO, Le Caire.

Depaule J.-C., « Vie quotidienne et espace habité au Machrek », Espaces des autres. Lectures anthropologiques d'architectures, Ed. de la Villette, coll. Penser l'espace, Paris, 1987.

Depaule J.-C., « Le Caire : emplois du temps, emplois de l'espace », Maghreb-Machrek n 127, La Documentation française, Paris, 1990.

Depaule J.-C., « Des espaces qualifiés : Présentation », Égypte/Monde arabe n5, $1^{\mathrm{er}}$ trim. 1991, Cedej.

Di Méo G., L'Homme, la Société, l'Espace, Anthropos-Economica, Paris, 1991.

El Kadi G., « La division sociale de l'espace au Caire : ségrégation et contradictions », MaghrebMachrek n 110, La Documentation française, Paris, 1985.

Ghitany G. (1989), Epître des destinées, Le Seuil, Paris, 1993.

Haqqi Y. (1944-1955), Choc, Denoël-Alif, Paris, 1991.

Ibrahim S. (1992), Les années de Zeth, Actes Sud, Paris. 1993.

Idris Y. (1957-1969), La sirène et autres nouvelles, Sindbad, Paris, 1986.

Ilbert R., « Le Caire a-t-il une médina ? ", Présent et avenir des Médinas (de Marrakech à Alep), Fascicule de Recherches n 10-11, Urbama, Tours, 1982.

Kohler L., «Instant diurne février 1988 : une tentative de représentation totale de la rue Gamaliyya », Égypte/Monde arabe n’ 5,1 ${ }^{\text {er }}$ trim., 1991, Cedej.

Krause R.-F., « Untersuchungen Zur Bazarstruktur von Kairo », Marburger Geographische Schiften n ${ }^{\circ}$ 99,Marburg, 1985.

Luizard P.-J., « Le soufisme égyptien contemporain », Égypte/Monde arabe n 2, 2 trim. 1990, Cedej.

Mahfouz N. (1956-1957), Impasse des Deux-Palais, 1985 ; Le Palais du désir, 1987 ; Le jardin du passé, 1989, J.-C. Lattès, coll. « Lettres arabes », Paris.

Mahfouz N., Récits de notre quartier, Sindbad, Paris, 1988.

Marthelot P., « Dimensions nouvelles d'une métropole : Le Caire », Revue géographique de l'Est, tome IX, $n^{\circ} 3-4,1969$.

Meinecke-Berg V., Meinecke M., « Preliminary Report on the UNESCO Survey of Al-Jamaliyya », Islamic Cairo: Architectural Conservation and Urban Development of tne Historic Center, Cairo, German Institute of Archaelogy, 1980,

Meyer G., « Manufacturing in Old Quarters of Central Cairo », Eléments sur les centres-villes dans le Monde arabe, Fascicule de Recherches n'19, Urbama, Tours, 1987.

Meyer G., « Economic and Social Change in the Old City of Cairo », communication présentée à la rencontre annuelle de la Middle East Studies Association of North America, San Antonio, 1990. 
Noweir S., Gaillard M.-N., Le Caire, tracés et empreintes, Ecole d'Architecture de Versailles, LADRHAUS, 1994.

Panerai P., Ammar L., Le Caire : observations sur le tissu urbain de la ville ancienne, Ecole d'Architecture de Versailles, LADRHAUS, 1991.

Raymond A., Le Caire, Fayard, Paris, 1993.

Stauth G., " Gamaleyya : What is it That There Remains. An Essay on "Popular Habitus" in a Cairean Quarter », Communication présentée au département de sociologie de l'Université de Durham, s.d.

Stauth G., « Gamâliyya : Informal Economy and Social Life in a Popular Ouarter of Cairo », Working Paper n ${ }^{\circ}$ 87, University of Bielefeld, Faculty of Sociology, 1986.

'Uways S., L'histoire que je porte sur mon dos : mémoires, Cedej, Le Caire, 1989.

Volait M., « Composition de la forme urbaine du Caire », Égypte. Recompositions, Peuples méditerranéens $n^{\circ}$ 41-42,1988.

Volait M., « Le Caire : les problèmes de la croissance à la lumière du recensement de 1986 ", Espace, Populations, Sociétés, 1988.

Zakariya M., "Complémentarités des espaces d'habitat et de travail dans la vieille ville du Caire », Politiques urbaines dans le Monde Arabe. Etudes sur le monde arabe $n^{\circ} 1$, Maison de l'Orient, Lyon, 1984.

\section{NOTES}

1. Je remercie Guy di Méo pour l'aide qu'il a bien voulu m'apporter.

2. C'est pour des raisons de politique éditoriale propres aux éditions Sindbad que le roman de Naguib Mahfouz, Awlad hâratina - littéralement, « les enfants de notre quartier » - a été publié en français en 1991 sous le titre Les fils de la médina.

3. Brunet R., Géographie universelle. Mondes nouveaux. Tome I, Le déchiffrement du monde, Hachette-Reclus, 1990.

4. Auriac F., Système économique et espace, Economica, Paris, 1983.

5. Voie principale de la ville fatimide, la Qasaba, orientée nord-sud et allant de Bâb alFutûh jusqu'à Bâb Zuwayla, est désignée comme rue al-Mu'izz li-din Allâh. Cet axe se poursuit jusqu'au cimetière sud. Nous utilisons ici ce terme ou l'appellation d'al-Mu'izz pour évoquer cet ensemble, bien qu'il porte des noms différents selon les secteurs.

6. Zuqâq, 'atfa et hâra désignent divers types de voies; le terme hâra peut en outre désigner un quartier et ses habitants.

7. Grand plan parcellaire du Caire réalisé à partir de la réactualisation du plan cadastral (au 500e) des années 1930 par l'Atelier du Caire, Ecole d'Architecture de Versailles.

8. Les qism sont divisés en shiyâkha et englobés dans le gouvernorat du Caire.

9. Orsenna E., L'exposition coloniale. Cité par Brunet R., Ferras R., Thery H., Les mots de la géographie, dictionnaire critique, Reclus/La Documentation française, coll. Dynamiques du territoire, Paris, 1992.

10. Indicateurs comme le pourcentage d'analphabètes, de célibataires, le nombre de personnes par pièce, le nombre d'enfants par femme, etc.

11. Ce secteur comptait environ 400.000 habitants en 1947 et 475.000 en 1960. 
12. D'une part, les catégories 1 et 2 du recensement (hommes d'affaires, professions libérales, PDG, enseignants du supérieur, etc.) et, d'autre part, les catégories 7, 8 et 9 (ouvriers des divers secteurs d'activités).

13. Ces qism (Bâb al-Sha'riyya, Gamâliyya, Darb al-Ahmâr, Mûskî el Khalîfa) regroupaient environ 480.000 habitants au recensement de 1986.

14. IAURIF-GOPP. Implementation of the Homogeneous Sector Concept. Homogeneous Sector $n^{\circ} 1$,Cairo Centre (2 vol.), Le Caire, 1988.

15. Camus A., Le premier homme, Gallimard, Paris, 1994

16. Ammar L, Charara M., Madœuf A., « Eléments pour une typologie des implantations contemporaines ", à paraître dans Établissements de rapport au Caire aux époques mamelouke, ottomane et contemporaine, IFAO, Le Caire.

17. Fêtes de l'anniversaire des saints, du Prophète ou des membres de sa famille (ahl albayt).

18. Chiffres donnés par le quotidien al-Ahrâm en 1994.

19. A l'occasion de la fêle d'al-Husayn, le train est gratuit pour les pèlerins.

20. J.-W. Mac Pherson recensait 45 mawlid dans la vieille ville et une quinzaine dans les cimetières qui la jouxtent, mais nombre d'entre eux ont aujourd'hui disparu. The moulids of Egypt (Egyptian Saints-Days), Le Caire, 1940.

21. Brunet R., "L'espace, règles du jeu », in Espaces, jeux et enjeux, Fayard, Paris, 1986. 22. Le dhikr est un rituel collectif pratiqué par les disciples soufis sous le contrôle du shaykh, qui consiste en la répétition des noms de Dieu selon des rythmes divers, et selon des gestuelles corporelles particulières.

23. Baladi signifie, littéralement « du pays », mais le concept renvoie à ce qui est égyptien, «traditionnel » et " populaire », Cf. Al-Messiri Nadim S., Ibn al-balad, a Concept of Egyptian Identity, Leyde, Brill, 1978.

24. En particulier le Mariott de Zamalek (hall Bayn al-qasrayn), le Sheraton Guezira (salle Zuqâq al-madâq) et le Ramsès Hilton.

25. Berque J., Al-Shakaa M., 1969.

26. Wast al-wast : littéralement : " centre du centre ».

27. Cités par Ammar L. et Charara M., 1991.

28. 4.718 étudiants étaient inscrits à l'Université d'al-Azhar pour l'année 92-93 (chiffres donnés par la Capmas, Statistical Year Book, Arab Republic of Egypt, 1994).

29. IAURIF-GOPP, Upgrading and Enhancing Central Districts of Cairo. North Gamalia Project (2 vol.). Objectives and Program ; Implementation File, Le Caire, 1990.

30. Becard L., Pages J.-L., « L'aménagement du Grand-Caire. Un projet ambitieux une coopération exemplaire », Les Cahiers de l'IAURIF n 75, Paris, IAURIF, 1985.

31. Charre A., "La mégapole est diabolique ", Mégapole, Espace civique et Design. Cahiers de l'Institut pour l'art et la ville, n 5-6, Givors, Maison du Rhône, Institut pour l'art et la ville, 1994.

32. Titre d'une nouvelle de Youssef IDRIS, cf. Battain T. et Labib A., 1991.

33. Roussillon A., La lutte contre les stupéfiants en Égypte. Enjeux sociaux d'une répression, Le Caire, Cedej, 1986.

34. Citées par Ammar L. et Charara M., 1991.

35. Les lieux centraux des quartiers chics et « intermédiaires » du Caire sont marqués, de Ma'âdi à Héliopolis, de Muhandisîn à Gîza en passant par Madînat Nasr, Zamalek et le centre-ville, par une litanie composite de Pizza Hut, Wimpy, La Poire, Baskin Robbin's, (et depuis peu Mac Donald's), pour la restauration, et de Mobacco, Benetton, New-Man et Kickers pour l'habillement (la liste n'est pas exhaustive). Un magasin de la 
chaîne de prêt-à-porter Benetton s'est implanté dans la ville ancienne mais a fermé récemment.

INDEX

Mots-clés : géographie, Le Caire, mawlid, espace, vieille ville

AUTEUR

ANNA MADCEUF

URBAMA 